\title{
ON THE EXISTENCE OF NORMAL MODE VIBRATIONS OF NONLINEAR SYSTEMS WITH TWO DEGREES OF FREEDOM*
}

\author{
BY \\ R. M. ROSENBERG \\ University of California, Berkeley, Cal.
}

\begin{abstract}
A nonlinear, conservative system having two degrees of freedom is considered. The potential energy is subject to certain restrictions which are consistent with the strain energy of springs. Then, the notion of transversals; i.e., of curves orthogonal to equipotential lines, is introduced in order to prove the existence of an in-phase and of an out-of-phase normal mode of vibration, no matter how nonlinear the springs. It is shown, that the transversals are also very helpful in the discussion of general motions of the system.
\end{abstract}

Introduction. In a series of earlier papers, the normal mode vibrations of nonlinear systems having many degrees of freedom have been discussed $[1,2,3,4,5]$. While it was possible to treat many properties of these normal mode vibrations, their existence was only established in the case of so-called "similar" normal mode vibrations** [3]. In this paper we should like to discuss the question of existence of normal mode vibrations when these are non-similar. The entire treatment here is restricted to systems having two degrees of freedom. Certain features of our proof are intimately linked with the fact that the number of degrees of freedom does not exceed two. Hence, it may be expected that the proof becomes more complicated for systems having more than two degrees of freedom.

The system. As a model of admissible systems, we consider a linear chain of three material points having respectively, masses $m_{0}=\infty, m_{1}$ and $m_{2},\left(0<m_{1,2}<\infty\right)$. Since $m_{0}$ is infinitely large, a force of finite magnitude cannot accelerate it; hence, it is a fixed point in an inertial reference frame. The masses $m_{1}$ and $m_{2}$ have, respectively, the degrees of translational freedom $u$ and $v$ in the direction of the chain. Each mass point is connected to the other two by a spring; thus, the entire system consists of two movable masses and three springs; two of the springs are so-called "anchor springs" since they terminate on a fixed point, and one is a so-called "coupling spring" since it couples the two masses together. The term spring identifies a mass-less, one-dimensional mechanical device which changes its length under the action of a force. It is capable of storing (but not of creating or destroying) energy, and the energy stored in it is assumed to be a continuous function of the absolute value of the length change and has continuous first and second derivatives. In consequence of this dependence on the absolute value of the length-change, every spring resists (or aids) a deflection of prescribed magnitude in the same degree, be it an extension or a compression of the spring.

The force required to produce a prescribed length-change in any spring is called the spring force of that spring. Let this length change be denoted by $w$. Then, the spring

*Received January 13, 1964.

**Normal mode vibrations are said to be "similar" when the wave shapes in any two degrees of freedom are similar, i.e. when they satisfy $x_{i}(t) / x_{j}(t) \equiv$ const. 
force of the $i$ th spring may be written as

$$
f_{i}(w)=A_{i} w+O\left(w^{2}\right)
$$

and, if $A_{i} \neq 0$, the quantity $O\left(w^{2}\right)$ is negligible compared to $A_{i} w$ for $|w| \ll 1$. We shall require that not all $A_{i},(i=1,2,3)$ be zero. Hence, nonlinearizeable systems [2, 3] are not included in the present treatment.

We assume that one, and only one, configuration of the system exists in which it is capable of remaining at rest indefinitely. This is the equilibrium position, and the origin of $u$ and $v$ is so chosen that $u=v=0$ in the equilibrium position. Finally, we assume that the spring forces are the only forces acting on the masses. The system described here is shown in two alternate forms in Fig. 1.
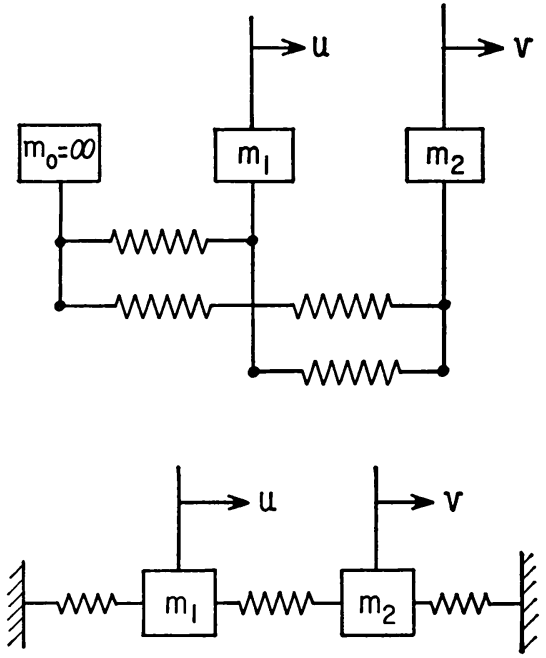

FIG. 1.

Representation of the motion. The system described here is conservative and scleronomous; hence the energy integral

$$
T-U=h
$$

exists where $T=T(\dot{u}, \dot{v})$ is the kinetic energy, $U(u, v)$ is a potential function equal to the negative of the strain energy stored in the springs, and $0<h=$ const. is the energy level of a given motion.

The mathematical representation of the motion may be made in two ways resulting, respectively, in the system $H$ (Hamilton) of equations, or in the system $M$ (Maupertuis). Hamilton's principle yields the equations of motion

$$
m_{1} \ddot{u}=\frac{\partial}{\partial u} U(u, v), \quad m_{2} \ddot{v}=\frac{\partial}{\partial v} U(u, v)
$$

and the transformations $x=m_{1}^{1 / 2} u, y=m_{2}^{1 / 2} v$ result in the system $H$

$$
\ddot{x}=\frac{\partial}{\partial x} U(x, y), \quad \ddot{y}=\frac{\partial}{\partial y} U(x, y)
$$

where dots denote differentiation with respect to time. 
The principle of least action (also called the principle of Maupertuis) leads to the system $M[4]$

$$
\begin{aligned}
& 2(U+h)\left\{x^{\prime \prime}\left(x^{\prime 2}+y^{\prime 2}\right)-x^{\prime}\left(x^{\prime} x^{\prime \prime}+y^{\prime} y^{\prime \prime}\right)\right\} \\
&+\left(x^{\prime 2}+y^{\prime 2}\right)\left\{x^{\prime}\left(x^{\prime} \frac{\partial U}{\partial x}+y^{\prime} \frac{\partial U}{\partial y}\right)-\left(x^{\prime 2}+y^{\prime 2}\right) \frac{\partial U}{\partial x}\right\}=0 \\
& 2(U+h)\left\{y^{\prime \prime}\left(x^{\prime 2}+y^{\prime 2}\right)-y^{\prime}\left(x^{\prime} x^{\prime \prime}+y^{\prime} y^{\prime \prime}\right)\right\} \\
&+\left(x^{\prime 2}+y^{\prime 2}\right)\left\{y^{\prime}\left(x^{\prime} \frac{\partial U}{\partial x}+y^{\prime} \frac{\partial U}{\partial y}\right)-\left({x^{\prime 2}}^{2}+y^{\prime 2}\right) \frac{\partial U}{\partial y}\right\}=0
\end{aligned}
$$

where primes denote differentiation with respect to the arc length $s$.

It is convenient to eliminate the parameter $s$ between the last pair of equations, because the form of the resulting single equation is much simpler than (3); one finds [1]

$$
2(U+h) y^{\prime \prime}+\left(1+y^{\prime 2}\right)\left(y^{\prime} \frac{\partial U}{\partial x}-\frac{\partial U}{\partial y}\right)=0,
$$

where, now, primes denote differentiation with respect to $x$. No confusion will arise with regard to this notation because the entire treatment which follows is based on (2) or (4). Hence, dots will always indicate differentiation with respect to time, and primes, differentiation with respect to $x$.

In some sense, (2) and (4) must be equivalent since they are both mathematical statements relating to the same problem. In fact, the equations of motion (2) have solutions $x(t), y(t)$; when $t$ is eliminated between them one finds $y=y(x)$ and this must satisfy (4).

Clearly, one may interpret (2) as the equations of motion of a unit mass, subjected to forces whose $x$ and $y$-components, respectively, are $U_{x}$ and $U_{\nu}$ (where subscripts are used to denote partial derivatives). This unit mass and force system is called the pseudosystem. During motion, the unit mass will trace out a trajectory $y(x)$ in the $x y$-plane and that trajectory satisfies (4). Hence, we may base future arguments either on the dynamical problem of the motion of the unit mass in the $x y$-plane, or on the geometric problem of integral curves of (4). Both viewpoints will be used.

Restrictions on $U$. The potential function $U(x, y)$ is subject to the following restrictions:

(a) it is negative definite;

(b) as mentioned earlier, it is a function of the absolute values of the length-changes of the springs, or

$$
U=U(|x|,|y|,|x-y|)
$$

(c) its partial derivatives are of the form

$$
\begin{aligned}
& U_{x}=a x+b y+P(x, y), \quad\left|\begin{array}{ll}
a & b \\
c & d
\end{array}\right| \neq 0 \\
& U_{y}=c x+d y+Q(x, y),
\end{aligned}
$$

where $P$ and $Q$ may be neglected compared with the leading terms in (6) when $|x|,|y| \ll 1$.

When $U(x, y)$ satisfies the conditions (a), (b) and (c), it is said to be admissible. 
An examination of the admissibility-conditions shows that they are consistent with the common concepts of springs and with the structural properties of materials from which springs are manufactured. Condition (a) implies that the spring forces tend to return the physical system to its equilibrium position. Condition (b) is equivalent to the observation that the spring forces are odd functions of the length-changes. Finally, condition (c) shows that, for sufficiently small length-changes, the springs behave like linear springs.

Normal mode vibrations. Here we shall define normal mode vibrations in terms of a vibration-in-unison of the physical system. An admissible spring-mass system is said to execute a vibration-in-unison if the motion satisfies all of the following conditions:

(i) all material points execute equi-periodic motion, or

$$
x_{i}(t)=x_{i}(t+T), \quad(i=1, \cdots, n)
$$

where $T$ is a constant;

(ii) there exists a time $t=t_{0}$ at which all particles pass through the equilibrium position, or

$$
x_{i}\left(t_{0}\right)=0, \quad(i=1, \cdots, n)
$$

(iii) there exists a time $t=t_{1} \neq t_{0}$ at which all velocities vanish, or

$$
\dot{x}_{i}\left(t_{1}\right)=0, \quad(i=1, \cdots, n)
$$

(iv) the position of every particle at any instant of time $t$ is uniquely determined by that of any one of them at the same instant, or

$$
x_{i}=x_{i}\left(x_{1}(t)\right), \quad(i=2, \cdots, n)
$$

are all single-valued functions of $x_{1}$.

In our system of two degrees of freedom, $x_{1}=x, x_{2}=y, i=1,2$.

If a linear system vibrates in normal modes, its motion satisfies all of the properties of a vibration-in-unison and conversely, when a linear system vibrates in unison, it moves in a normal vibration mode. Hence, it is reasonable to identify "vibration-inunison" with "normal-mode-vibration" whether the system is linear, or nonlinear. In this paper we shall prove the following central theorem.

Theorem. A system of two degrees of freedom whose equations of motion are (4) possesses at least two normal mode vibrations in the sense of conditions ( $i)$ to $(i v)$, if $U(x, y)$ is admissible in the sense of restrictions (a) to (c).

The geometry of normal mode vibrations. Before proceeding to the proof of the above theorem, it is helpful to interpret vibration-in-unison in terms of trajectories in the $x y$-plane; i.e., of integral curves of (4). When condition (iii) is satisfied, the kinetic energy $T\left(\dot{x}\left(t_{1}\right), \dot{y}\left(t_{1}\right)\right)=0$ because $T$ is a positive definite, quadratic form in the velocities. Then, the energy integral (1) reduces at $t=t_{1}$ to

$$
U(X, Y)+h=0, \quad h>0
$$

where $(X, Y)$ are those points in the $x y$-plane for which the velocity of the unit mass (of the pseudo-system) vanishes when the energy level is $h$. Equation (7) defines a curve in the $x y$-plane called the $\Gamma$-curve. In view of the admissibility conditions (a) and (b), the $\Gamma$-curve is closed, surrounds the origin and is symmetric with respect to it. This curve is also called the bounding curve because it has been shown $[1,4]$ that all 
integral curves of (4) must lie in the finite, closed domain bounded by the $\Gamma$-curve. Since condition (iii) is satisfied by a normal mode vibration, every integral curve of (4) which corresponds to a normal mode vibration must intercept the $\Gamma$-curve.

Since a normal mode vibration satisfies condition (ii), $x$ and $y$ vanish simultaneously or, every integral curve of (4) which corresponds to a normal mode vibration must pass through the origin of the xy-plane.

Finally, (iv) is satisfied or, every integral curve $y(x)$ of (4) which corresponds to a normal mode vibration must be single-valued. An integral curve which satisfies all these properties is called a modal line, and is denoted by $T^{*}$ or $T^{* *}$.

Clearly, the converse of the above statements is also true, or every integral curve of (4) which has the above three properties corresponds to a normal mode vibration. Hence, our theorem will be proved if we can show that there exist two integral curves of (4) which originate on the outer contour, which pass through the origin and which are single-valued. In this proof, we shall use the following theorem.

TheOREM. Every integral curve of (4) which intercepts the bounding curve intersects it orthogonally $[1,4]$.

The transversals. In order to prove the existence theorem, it is helpful to introduce the notion of the transversals. The equations

$$
U(x, \varphi)+h^{*}=0,
$$

where $h^{*}$ is a constant satisfying $0<h^{*} \leq h$, define the equipotential curves $y=\varphi(x)$, called henceforth " $E$-curves". Inasmuch as $U$ is negative, definite and $h^{*}>0$, the $E$-curves constitute a one-parameter family of non-intersecting closed curves surrounding the origin, and they satisfy

$$
\frac{d \varphi}{d x}=-\frac{U_{x}(x, \varphi)}{U_{\varphi}(x, \varphi)},
$$

as may be readily verified by differentiating (8). The transversals are the family of curves which intersect the equipotential curves orthogonally. We shall call them the $P$-curves and denote them by $y=\theta(x)$. In view of (9) they satisfy

$$
\frac{d \theta}{d x}=\frac{U_{\theta}(x, \theta)}{U_{x}(x, \theta)} .
$$

The transversals have a property which is of central importance to the problem in hand: the P-curves define the direction field of the forces which act on the unit mass of the pseudo-system.

This will be seen from an inspection of the equations of motion of the pseudo-system; i.e. from (2). Since the right hand sides of (2) are the force components of the pseudosystem it is clear that the $P$-curve which passes through any point $(x, y)$ does so with the slope of the force of the pseudo-system. The $P$-curves connect points on the $\Gamma$ curve with the origin. We shall endow them with a sense of direction by defining their intersection with the $\Gamma$-curve as their "point of issue." Then, their sense of positive direction is such as to point toward the origin of the $x y$-plane. Their importance to our problem is that the forces of the pseudo-system are tangent to, and point in the positive direction of the $P$-curves, and they decrease monotonically to zero as the $P$-curve tends to the origin. Because of this property, it is desirable to explore the $P$ curves in some detail. 
It was already observed that (8) describes a continuum of closed curves surrounding the origin, and every neighborhood containing the origin in its interior also contains such curves. Moreover, since $U$ is a definite form, $U_{x}$ and $U_{y}$ vanish simultaneously at the origin. Hence, (9) has a singular point at the origin, and this singularity must be a center. Now, the Poincare theory of simple singular points [6] shows that the character of the singular point of a differential equation of the form

$$
\frac{d y}{d x}=\frac{c x+d y}{a x+b y}, \quad\left|\begin{array}{ll}
a & b \\
c & d
\end{array}\right| \neq 0
$$

can be deduced from certain relations between the constants. The cases of interest here are the following:

(I) if $a+d=0$ and $a d-b c>0$, the origin is a center;

(II) if $a+d \neq 0, a d-b c>0$ and $(a+d)^{2}-4(a d-b c)>0$, the origin is a node. From the established fact that the $E$-curves form a center at the origin, we shall now deduce the the origin is a node for the transversals.

Expanding the partial derivatives of $U$ about the origin one has, since $U_{x}(0,0)=$ $U_{\nu}(0,0)=0$,

$$
\begin{aligned}
& U_{x}=x U_{x x}+y U_{x y}+\text { higher order terms } \\
& U_{y}=x U_{y x}+y U_{y y}+\text { higher order terms }
\end{aligned}
$$

If these are introduced into the equation of the $E$-curves; i.e., into (9), one finds that. for the equipotential curves

$$
a=U_{x y}, \quad b=U_{y y}, \quad c=-U_{x x}, \quad d=-U_{x y} .
$$

Then, it is seen that $a+d=0$, and

$$
a d-b o=U_{x x} U_{y y}-U_{x y}^{2} .
$$

Hence, the first condition of (I) is satisfied and, from the second it follows that, in the neighborhood of the origin,

$$
U_{x x} U_{y y}-U_{x y}^{2}>0 .
$$

Since the origin is a center for the $E$-curves, and since every $P$-curve must intersect every $E$-curve, it follows that every $P$-curve must pass through the origin. But the $P$-curves satisfy (10), and that equation also has a singular point at the origin. Hence the origin must be either a node or a focus for the $P$-curves because these are the only simple singular points that are intersected by every integral curve.

The substitution of (11) and (10) shows that, for the transversals,

$$
a=U_{x x}, \quad b=U_{x y}, \quad c=U_{x y}, \quad d=U_{y y} .
$$

Hence, for the $P$-curves,

$$
a+d=U_{x x}+U_{y y},
$$

which does not vanish, in general. The quantity

$$
(a+d)^{2}-4(a d-b c)=\left(U_{x x}-U_{y y}\right)^{2}+4 U_{x y}^{2},
$$

which is positive, and finally one has 


$$
a d-b c=U_{x x} U_{y y}-U_{x y}^{2} .
$$

But, in view of (12), that quantity is also positive and, thus, all conditions (II) are satisfied. It follows, that the origin is a node for the transversals, which proves the following lemma.

Lemma 1. All P-curves pass through the origin with common slope $\theta_{0}^{\prime}$.

Consider the $x y$-plane shown in Figure 2 together with the $\Gamma$-curve. As stated earlier, the $\Gamma$-curve is the locus of points $P(X, Y)$ of zero velocity for energy level $h$. Let the point $P_{0}\left(X_{0}, Y_{0}\right)$ denote the intersection of the $\Gamma$-curve with the straight line which passes with slope $\theta_{0}^{\prime}$ through the origin. Further, let $P_{1}\left(X_{1}, Y_{1}\right)$ be a point on the $\Gamma$ curve whose distance from the origin is stationary with respect to neighboring points on the $\Gamma$-curve. In Figure 2, the point $P_{0}$ is shown "below" $P_{1}$. No generality is lost thereby because an exchange of $x$ and $y$-axes can always produce this configuration. Moreover, no generality is lost by locating both of these points in the first quadrant. We simply designate as the first quadrant that which contains $P_{0}$. Since every quadrant contains at least one point on the $\Gamma$-curve which has stationary distance from the origin, we can always produce the configuration shown. We expressly except the case of coincidence of $P_{0}$ and $P_{1}$ because that case can be shown to result in so-called "similar" normal mode vibrations, a case which is very much simpler that the one discussed here and which has been treated elsewhere $[2,3,4,5]$.

Now, the normal to the $\Gamma$-curve at the point $P_{1}$ points in the direction of the origin. Hence, the transversal issuing from $P_{1}$ must have a point of inflection in order to terminate at the origin with slope $\theta_{0}^{\prime}$.

Similarly, the normal to the $\Gamma$-curve at the point $P_{0}$ points "above" the origin. Consequently, it must also have a point of inflection in the first quadrant as it also terminates at the origin with slope $\theta_{0}^{\prime}$.

It is seen, then, that at least some transversals have points of inflection. The locus of points of inflection of $P$-curves is readily found by equating to zero the second derivative of $\theta$ with respect to $x$. By differentiating (10), one finds

$$
\theta^{\prime \prime}=\frac{U_{x \theta}\left(U_{x}^{2}-U_{\theta}^{2}\right)-U_{x} U_{\theta}\left(U_{x x}-U_{\theta \theta}\right)}{U_{x}^{3}},
$$

and the locus of inflection points of $P$-curves is given by

$$
F(x, y)=U_{x y}\left(U_{x}^{2}-U_{y}^{2}\right)-U_{x} U_{y}\left(U_{x x}-U_{y y}\right)=0 .
$$

We shall call this locus the $F$-curve; it is also shown in Figure 2.

The $F$-curve must necessarily intersect the transversals issuing from $P_{0}$ and $P_{1}$ because both have been shown to have inflection points. The intersection of the $F-$ curve with the $\Gamma$-curve is denoted as the point $P_{2}\left(X_{2}, Y_{2}\right)$. One can readily show that the $F$-curve enters the origin with slope $\theta_{0}^{\prime}$ and, there it has second-degree contact with the straight line connecting the origin and $P_{0}$.

For later purposes, it is also of interest to construct the locus of points on $E$-curves whose distance from the origin is stationary with respect to neighboring points on the same $E$-curves. It is easy to show that this locus is defined by the equation

$$
G(x, y)=y U_{x}-x U_{y}=0 .
$$

This locus will be called the $G$-curve; it intersects the $\Gamma$-curve at $P_{1}$, and it enters the 


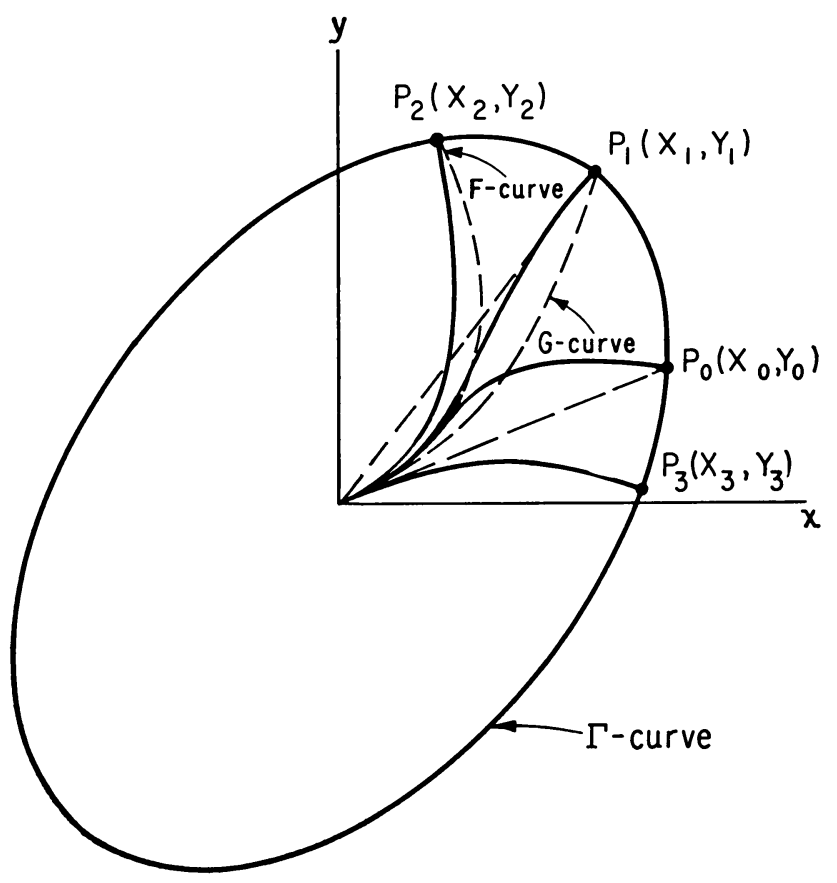

FIG. 2.

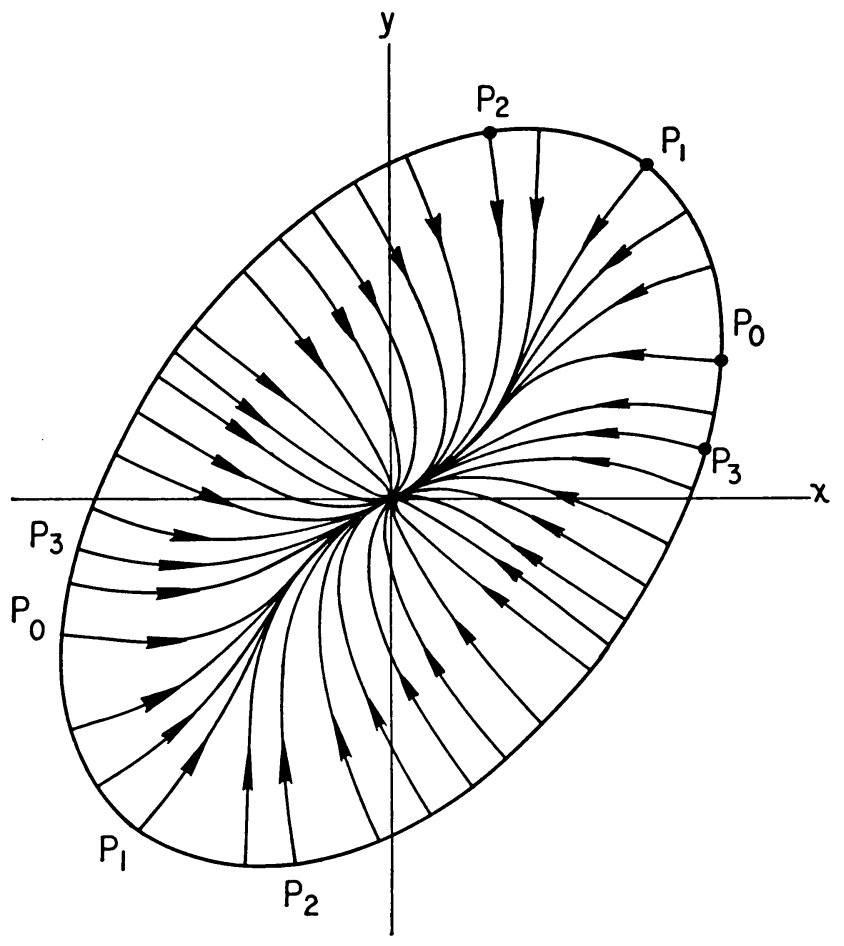

Fig. 3. 
origin with slope $\theta_{0}^{\prime}$ and has second degree contact there with the straight line from the origin to $P_{0}$.

Consider now the transversals as the point of issue moves in a clockwise direction from the positive $y$-axis intercept to the positive $x$-axis intercept along the $\Gamma$-curve. The initial curvature starts out being positive, say, and decreases monotonically to zero at $P_{2}$; it then becomes negative. The transversals beyond $P_{2}$ will have points of inflection until the point of issue has descended to some point $P_{3}\left(X_{3}, Y_{3}\right)$ which is such that the transversal issuing from it has second degree contact at the origin with the straight line between the origin and $P_{0}$. Transversals issuing from a point below $P_{3}$ have no intersection with the $F$-curve and, hence, no point of inflection.

Inasmuch as $U$ is symmetric with respect to the $U$-axis in the $x, y, U$-space, every locus and transversal in Fig. 2 is symmetric with respect to the origin of the $x y$-plane. Hence, the entire field of $P$-curves can now be constructed; it is shown in Fig. 3. In this figure, arrows have been placed on the transversals, indicating their positive directions.

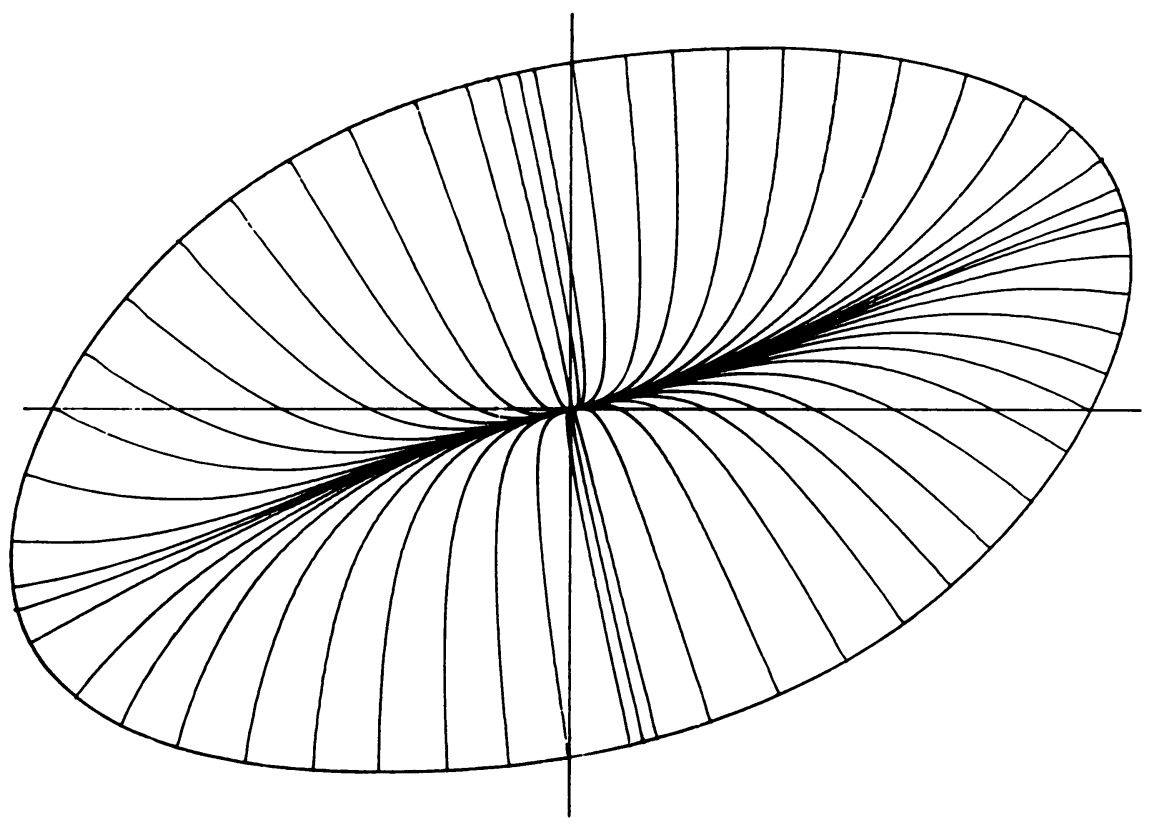

Fig. 4.

Figure 4 is a photograph of a set of curves drawn entirely by an analog computer. The $\Gamma$-curve was produced by letting the computer integrate (9) at the energy level $h$. The transversals were obtained by letting the analog computer integrate (10) for initial points lying on the $\Gamma$-curve. The particular example, solved by the computer was

$$
U=-\frac{1}{2} x^{2}-\frac{1}{4} x^{4}-\frac{5}{2} y^{2}-\frac{5}{4} y^{4}-\frac{5}{2}(x-y)^{2}-\frac{5}{4}(x-y)^{4}, \quad h=1 .
$$

This particular $U$ was chosen to insure that the system is strongly nonlinear, and that it is not nearly "symmetric". (A symmetric system is one in which the anchor springs, are equal and the masses $m_{1}$ and $m_{2}$ are equal, and it always has similar normal mode 
vibrations.) It is seen that the experimental results agree in every detail with theoretical predictions.

The trajectories. We shall now consider certain trajectories of the unit mass of the pseudo-system; i.e., certain integral curves of (4). The trajectories considered are those which issue from points on the $\Gamma$-curve; they will be called $T$-curves. The transversal which issues from the same point on the $\Gamma$-curve as a given trajectory will be called the transversal associated with that trajectory, or the "associated P-curve."

For our purposes, it is convenient to introduce (10) in (4), and to rewrite the latter as

$$
y^{\prime \prime}=-\frac{\left(1+y^{\prime 2}\right)}{2(U+h)} U_{x}\left(y^{\prime}-\theta^{\prime}\right) \text {. }
$$

Now, the equation (10) of the transversals $\theta(x)$ is singular at the origin, and the equation (16) of the trajectories is singular on the $\Gamma$-curve (that curve being defined by $U+h=0)$. Therefore, we shall denote as regular points all those, except the origin, which lie inside of, but not on, the $\Gamma$-curve.

We shall now establish a number of properties of the trajectories issuing from points $P(X, Y)$ of the $\Gamma$-curve. First we state a lemma.

Lemma 2. Every trajectory is tangent to its associated transversal on the $\Gamma$-curve. Evidently, this requires that trajectories intercept the $\Gamma$-curve orthogonally, and this has been proved elsewhere $[1,4]$.

Lemma 3. The curvature $\kappa_{T}(X, Y)$ of a trajectory at its point of issue $P(X, Y)$ has the same sign, but is less in magnitude, than the initial curvature $\kappa_{P}(X, Y)$ of its associated transversal.

To prove this lemma, we first note that the curvature of a $T$-curve at its point of issue is given by [5]

$$
y^{\prime \prime}(X, Y)=\frac{1}{3}\left[\frac{U_{x y}+y^{\prime}\left(U_{y y}-U_{x x}-y^{\prime} U_{x y}\right)}{U_{x}}\right]_{X, Y} .
$$

But, in view of Lemma 2,

$$
y^{\prime}(X, Y)=\frac{U_{y}(X, Y)}{U_{x}(X, Y)}
$$

If this relation is introduced in (17), one finds

$$
y^{\prime \prime}(X, Y)=\frac{1}{3}\left[\frac{U_{x y}\left(U_{x}^{2}-U_{y}^{2}\right)-U_{x} U_{y}\left(U_{x x}-U_{y y}\right)}{U_{x}^{3}}\right]_{X, Y} .
$$

A comparison between (13) and (19) shows that

$$
\kappa_{T}(X, Y)=\frac{1}{3} \kappa_{P}(X, Y),
$$

which proves Lemma 3. It states essentially that, at its point of issue, a trajectory is "bent in the same direction, but less so" than its associated transversal.

Next we prove the following lemma.

Lemma 4. No trajectory can coincide everywhere with its associated transversal unless that transversal is a straight line.

For the proof, we observe from the equations of motion (4) that a mass point, acted on by a force having components $U_{x}$ and $U_{y}$, tends to follow a curved transversal as its mass tends to zero and follows it precisely only in the case of zero mass. However, 
the mass of the pseudo-system is of magnitude unity, not zero, which proves Lemma 4.

LEMma 5. At any regular point, the curvature of every trajectory is such as to yield to the force field.

From the standpoint of predicting the behavior of a trajectory that passes with prescribed slope through a given point, Lemma 5 is the most useful of all those given here. To prove it consider Fig. 5 showing a family of transversals, and a trajectory

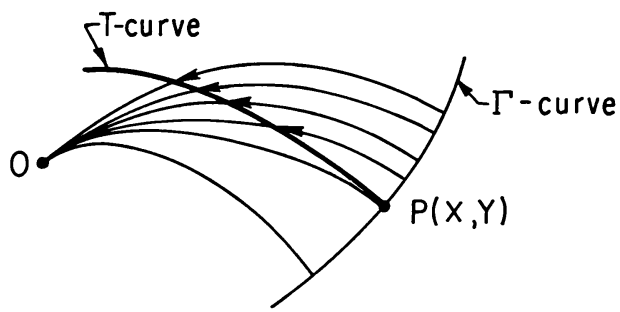

FIG. 5 .

$T$ intersecting them. (Note that every trajectory intersects or is tangent to one transversal at every regular point, because the transversals fill out the entire domain inside the $\Gamma$-curve, and no curved trajectory coincides with any of them.) The arrows on the transversals indicate the direction of the forces acting on the unit mass at every point. It is evident (without a detailed proof that could easily be constructed by considering force components tangent and normal to the trajectory) that Lemma 5 is true.

LEMma 6. If a trajectory is tangent to a transversal at any regular point, it has zero curvature at that point.

The proof of this lemma follows directly from (16). This property of trajectories was noted earlier by Kauderer [7].

Lemma 7. Every trajectory that intersects its associated transversal at a regular point must have passed through a point of zero curvature at some regular point.

For the proof of this lemma consider Fig. 6 which shows a trajectory that intersects

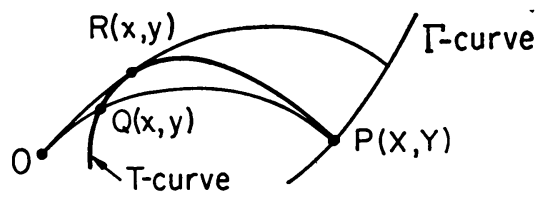

FIG. 6.

its associated transversal. Clearly, there exists, then, some other transversal which is tangent to the trajectory at some intermediate point $R(x, y)$ between the point of issue $P(X, Y)$ and the point of intersection $Q(x, y)$. In view of Lemma $6, R(x, y)$ is a point of zero curvature of the trajectory.

Lemma 8. Every trajectory that does not pass through the origin must have at least one point of tangency with at least one equipotential line.

Note that this does not exclude the possibility that the trajectory may pass through the origin after tangency with an equipotential line. The proof of this lemma is selfevident; however, a formal proof can be made on the basis that every integral of (4) 

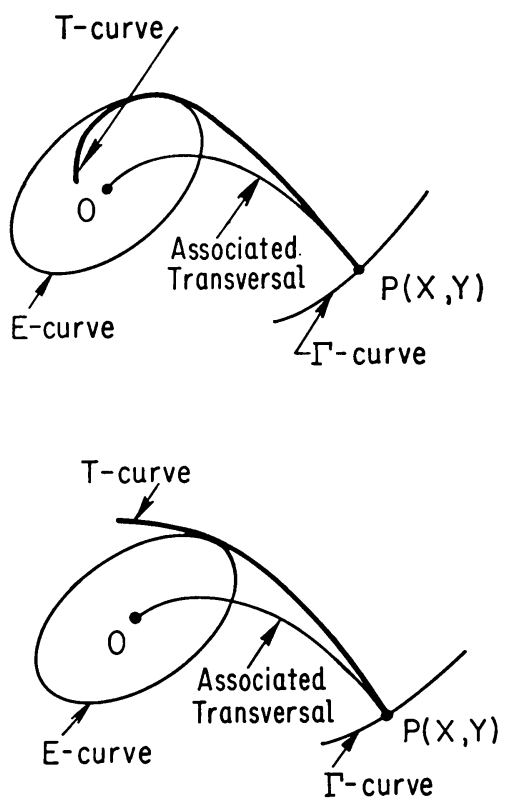

FIG. 7.

is a continuous smooth curve that must lie in the finite closed domain bounded by the $\Gamma$-curve. This lemma is illustrated in Fig. 7.

Lemma 9. No trajectory can have a point of zero curvature prior to its first point of tangency with an equipotential line.

This is the central lemma required for our proof of the basic theorem. To prove it, we make use of the dynamics of the pseudo-system. We first observe that vanishing of the trajectory curvature at a regular point $Q(x, y)$ implies, in general, that $Q$ is a point of inflection of the trajectory because, in general, $y^{\prime \prime \prime}(x)$ does not vanish at the same point as $y^{\prime \prime}(x)$. However, let us assume that both these derivatives do vanish at the same point. Then, there exist some trajectories $\bar{y}(x)$ neighboring on $y(x)$, for which $\bar{y}^{\prime \prime}(x)=0$ at some point $\bar{Q}(\bar{x}, \bar{y})$ in the neighborhood of $Q$. For this trajectory, $\bar{y}^{\prime \prime \prime}(x)$ will certainly not vanish where $\bar{y}^{\prime \prime}(x)$ vanishes. Now consider Figs. 8(A) and (B), both showing trajectories which have a point of inflection at $Q$. The difference between these is that the curvatures of the transversals in the neighborhood of $Q$ have, in Case (A), curvatures of the same sign as that of the trajectory prior to its arrival at $Q$, and in Case (B), these curvatures are opposite in sign. Clearly, Case (A) contradicts Lemma 5 and is, thus, ruled out.

Now, consider Figs. $8(\mathrm{C})$ and (D). The difference in these is connected with the direction of the forces as the transversals intersect the trajectory. In Case (C), the forces change direction such that the force direction at $Q$ is reached monotonically while, in Case (D), the force direction "overshoots" and then returns. In other words, the angle between the force direction at $Q$ and at any point on the trajectory prior to its arrival at $Q$ has, in Case (C), only a single zero at $Q$ and, in Case (D), it also has a zero at some point on the trajectory prior to its arrival at $Q$. We shall show that Case (C) is excluded. For, assume that the force direction had had, everywhere along the 
trajectory, the direction which it has at $Q$. Such a case would certainly be more favorable to producing tangency between force and trajectory at $Q$ than Case $(\mathrm{C})$ which is being considered. However, if the initial angle between velocity and a force of constant direction and finite magnitude is not zero, then it requires an infinitely long time to reduce that angle to zero. Hence, Case (C) cannot arise.

We now consider Case (D). Consider the angle between trajectory and force direction measured from trajectory to the force direction. This angle is, initially, acute and, near $Q$, it is obtuse. A simple consideration shows that this statement is true for any trajectory which satisfies Lemma $\mathbf{5}$ and which is tangent to a transversal at some regular point. But, if it holds, than there must be at least one point on such a trajectory prior to its arrival at $Q$ where the force direction is normal to the trajectory. Then, since the forces are tangent to the transversals, and the equipotential lines are normal to them, the trajectory must be tangent to an equipotential line prior to its arrival at $Q$. This proves Lemma 9 .

We require one final property in order to prove the following basic lemma.

LEMma 10. If a trajectory $y=f(x)$ which passes through the origin has prior tangency with an equipotential line, it cannot be a modal line.

The condition (iv) of normal mode vibrations, relating to single-valuedness requires that the modal line $y=f^{*}(x)$ possess everywhere an inverse $x=g^{*}(y)$, or the relation

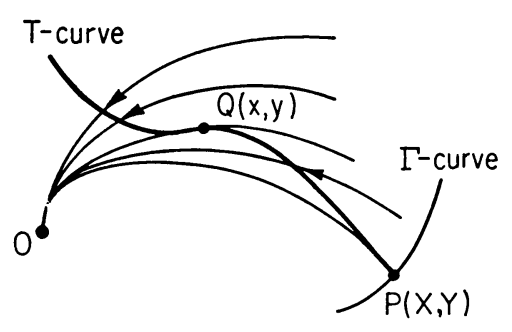

(A)

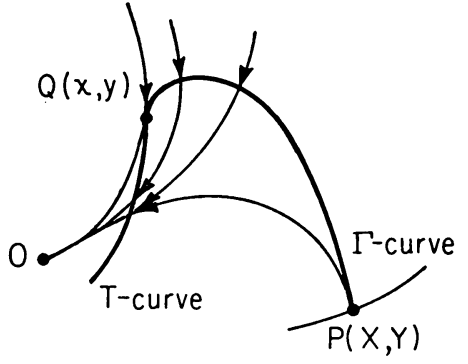

(B)

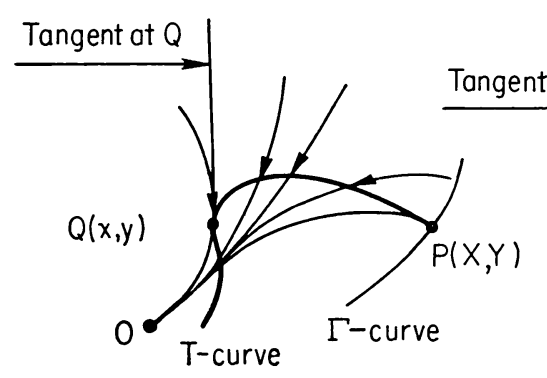

(C)

Fig. 8. 


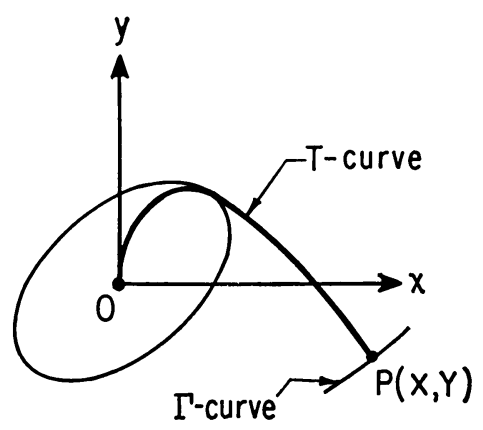

Fig. 9.

between $f_{1}^{*}, g^{*}$ on the one hand and $x, y$ on the other must be a homeomorphism. Consider now Figure 9 which illustrates a trajectory that attains the origin after having been tangent to an $E$-curve. It has been drawn in such a fashion that $y$ is a single-valued function of $x$. However, it is clear that now, $x$ is no longer a single-valued function of $y$. A simple consideration shows that no case can be constructed where a trajectory, satisfying Lemma 5, violates Lemma 10.

While the above properties of trajectories provide the material with which to prove the basic theorem, it is evident that they are also useful in a general discussion of integral curves of (4), particularly if these integral curves attain the $\Gamma$-curve.

The existence of modal lines. We proceed now with the proof of the basic theorem. First, we observe that every trajectory which issues from the $\Gamma$-curve does so with a prescribed slope; i.e., normal to the $\Gamma$-curve. Now, the equation whose integrals we are discussing; i.e., (4), satisfies the conditions for existence and uniqueness of solutions everywhere in the open region that contains the origin and is bounded by the $\Gamma$-curve; hence, not two distinct solutions can issue from the same point on the $\Gamma$-curve. It follows that no trajectory issuing from the $\Gamma$-curve can be a closed curve. By Lemmas 8 and 9 , every trajectory, except the modal line, is tangent to some equipotential line without having had a point of inflection prior to this occurrence. Moreover, by Lemma 10, the trajectory is not a modal line if its passage through the origin is preceded by tangency with an $E$-curve. Hence, we have the following theorem.

Theorem 1. Modal lines, if they exist, of a two-degree-of-freedom system with admissible $U$ in the sense of conditions $(i)$ to $(i v)$ do not have points of inflection.

This theorem proves that no modal line can issue, in the first quadrant, from points on the arc $\left(P_{2} P_{1}\right]$ of the $\Gamma$-curve (see Fig. 2) because any trajectory issuing from such points cannot pass through the origin without having at least one point of inflection. This is a consequence of the fact that trajectories issuing from these points not only point to the right of the origin but have initial curvature moving them still further to the right.

It is now evident that the search for modal lines consists in seeking trajectories that issue from the $\Gamma$-curve and which are "tangent to the equipotential line of zero energy"; i.e., the origin, without having been, earlier, tangent to some other equipotential line.

The general idea of our proof is illustrated in Fig. 10. We shall consider two trajectories $T_{0}$ and $T_{2}$ issuing, respectively, from points $P_{0}\left(X_{0}, Y_{0}\right)$ and $P_{2}\left(X_{2}, Y_{2}\right)$ and 
demonstrate that $T_{0}$ passes above the origin, and $T_{2}$ to the right of it. Hence there must exist a point $P^{*}\left(X^{*}, Y^{*}\right)$ lying between $P_{2}$ and $P_{0}$ such that the trajectory $T^{*}$ passes through the origin. This is a consequence of the fact that the differential equation (4), satisfied by any $T$-curve, is regular in the open region bounded by the $\Gamma$-curve and, hence, the solution depends in a continuous manner on the initial condition. In other words, as the point of issue $P(X, Y)$ moves in a continuous manner in a counterclockwise direction from $P_{0}$ toward $P_{2}$, the $y$-axis intercept of the $T$-curve moves in a continuous manner toward the origin.

Consider the $T_{0}$-curve which issues from $P_{0}\left(X_{0}, Y_{0}\right)$. By Lemma 2, it is tangent at $P_{0}$ to its associated trajectory. By Lemma 3 it has a lesser initial curvature, but of the same sign, as its associated trajectory. Hence, it lies at first above its associated trajectory. Now, this curve either passes through the origin in which case it is the desired $T^{*}$-curve, or it is by Lemma 9 tangent to an $E$-curve without having had a prior point of zero curvature. But, then, by Lemma 7, it cannot have crossed its associated transversal and must, thus, have remained above it, as shown.

Precisely the same arguments show that the $T_{2}$-curve must have remained to the right of its associated trajectory and, thus, passes to the right of the origin.

Now consider a trajectory issuing from a point $P(X, Y)$ as that point moves in a continuous fashion in a counter-clockwise direction from $P_{0}$ to $P_{2}$. The resulting $T$ curve which, initially, coincides with the $T_{0}$-curve now changes in a continuous fashion until it coincides with the $T_{2}$-curve. Hence, it must have passed through a point $P^{*}\left(X^{*}, Y^{*}\right)$ at which it has passed through the origin. This is the modal line, or the $T^{*}$-curve.

It is of interest to note that the point $P^{*}$ must be interior to the $\operatorname{arc}\left(P_{0} P_{1}\right]$ because,

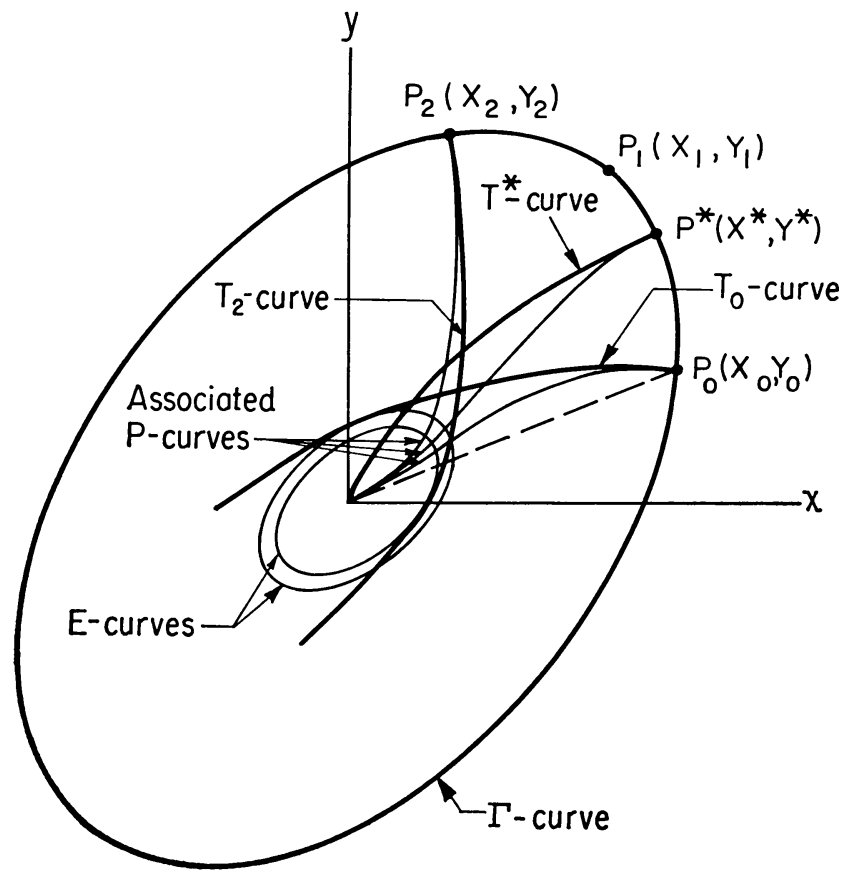

Frg. 10. 


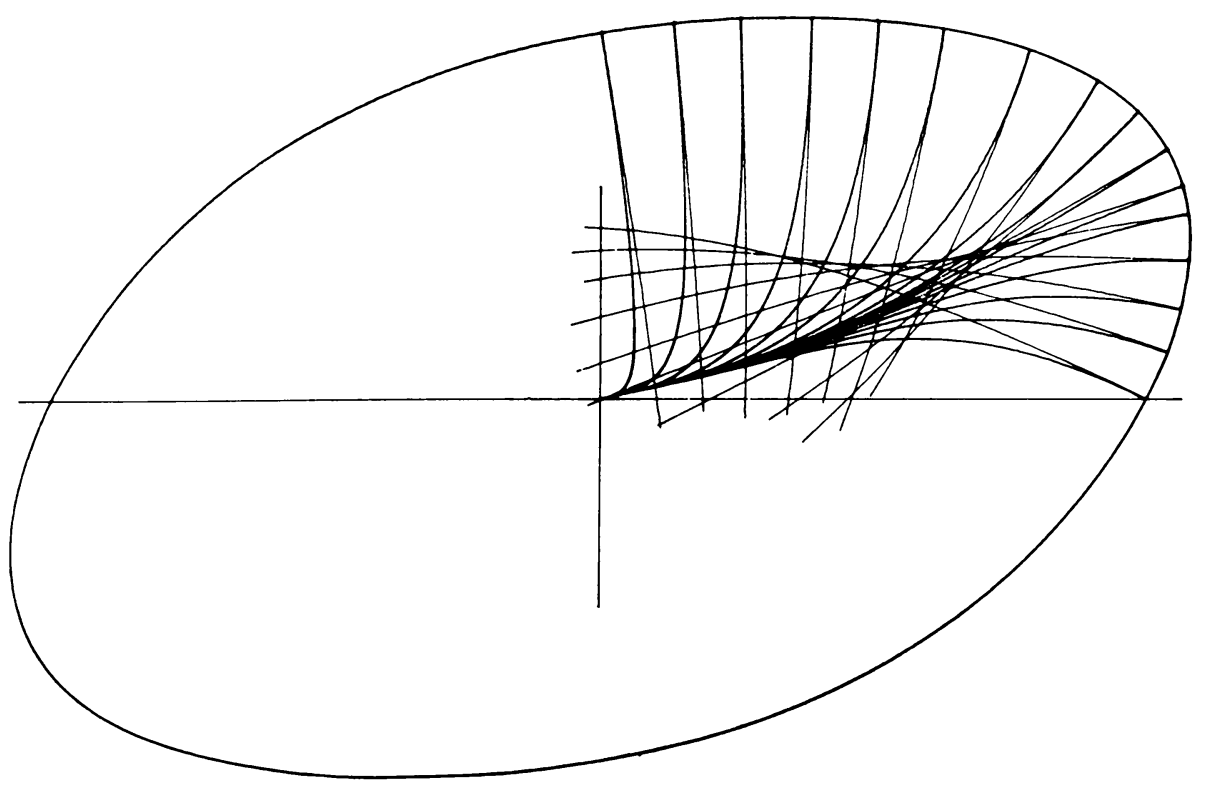

FIG. 11.

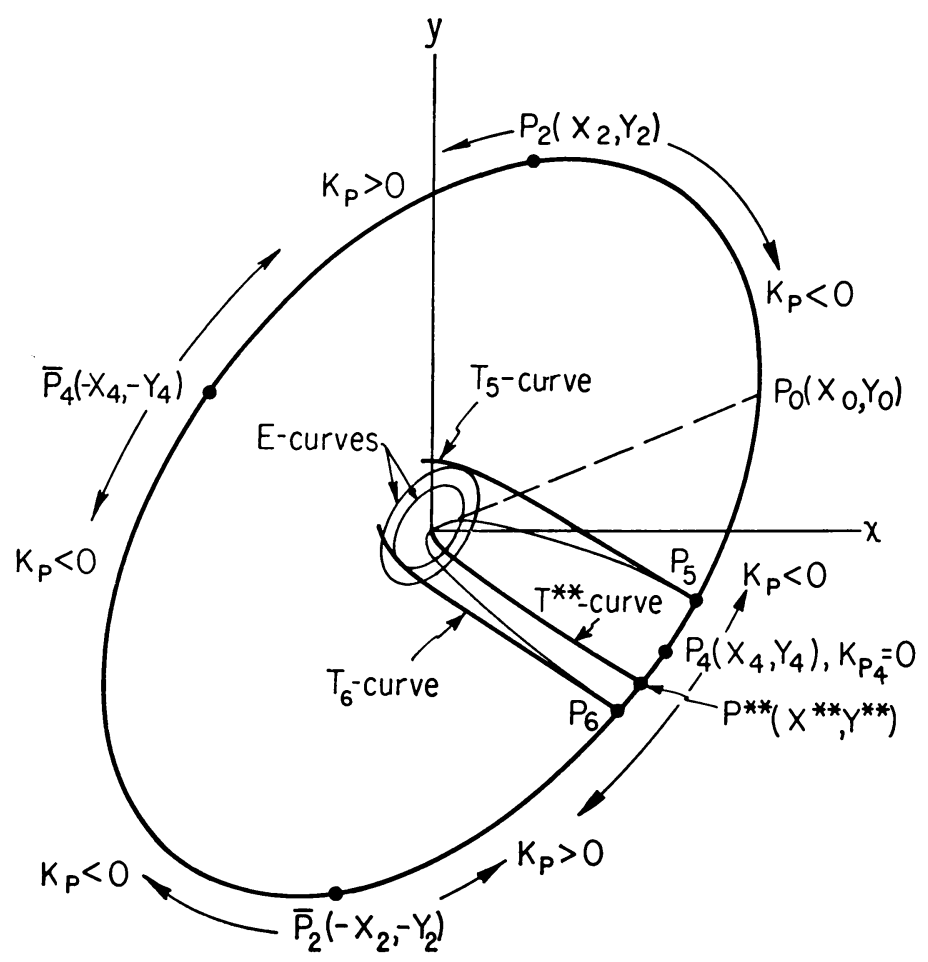

Fig. 12. 
by Theorem 1 no modal line can originate on $\left(P_{2} P_{1}\right]$. Moreover, the curvature of this modal line is negative because the initial curvatures of all transversals in the interval $\left(P_{2} P_{1}\right]$ are negative, and there is no change of curvature. Finally, this modal line gives rise to an in-phase normal mode vibration because it lies in the first quadrant so that $y>0$ when $x>0$. Hence, we have the following theorem.

Theorem 2. A system of two degrees of freedom whose equations of motion are (4) possesses an in-phase normal-mode-vibration in the sense of conditions (i) to (iv), if $U(x, y)$ is admissible in the sense of restrictions (a) to (c).

In Fig. 11 is shown a photograph of a set of curves drawn entirely by an analog computer. The $\Gamma$-curve was traced by the computer which integrated (9) under initial conditions $h^{*}=h$. Also shown is a family of trajectories which were drawn by the computer by integrating (4), and associated transversals by integrating (10). The numerical values were the same as those given in connection with Fig. 4. It is seen that the experimental results bear out entirely the theory presented here.

We now proceed to the second part of the basic theorem. Consider Fig. 12 showing the $\Gamma$-curve and the point $P_{2}\left(X_{2}, Y_{2}\right)$ where the initial curvature of the transversals vanishes, being positive to the right of $P_{2}$ and negative to the left. Now because of the symmetry of the entire figure with respect to the origin, there exists a point $\bar{P}_{2}\left(-X_{2},-Y_{2}\right)$ in the third quadrant where $\kappa_{P}(X, Y)$ vanishes also and, (by circular symmetry also) $\kappa_{P}(X, Y)$ is positive to the right of $\bar{P}_{2}$ and negative to the left. Next, consider either arc $\left(P_{2} \bar{P}_{2}\right)$ on the $\Gamma$-curve. It will be seen that at one end of this arc $\kappa_{P}(X, Y)>0$, and at the other $\kappa_{P}(X, Y)<0$. Since the initial curvature changes in a continuous manner with a continuous change of the point $P(X, Y)$ on the $\Gamma$-curve, there must be at least one point on the arc $\left(P_{2} \bar{P}_{2}\right)$ where the curvature also vanishes. Let it be denoted by $P_{4}\left(X_{4}, Y_{4}\right)$. Then, by circular symmetry, $\kappa_{P}$, also vanishes at

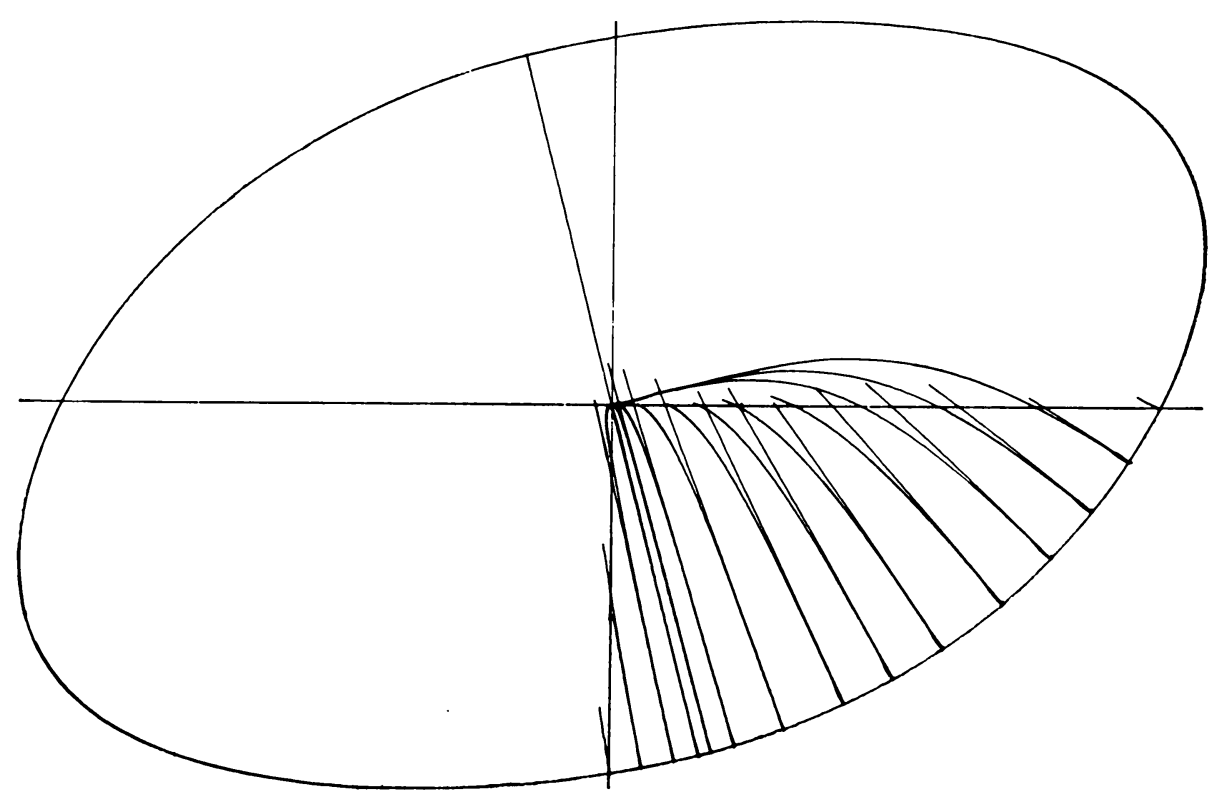

Fig. 13. 
$\bar{P}_{4}\left(-X_{4},-Y_{4}\right)$, and the transversals issuing from either side of $P_{4}$ have opposite initial curvature. This is, in fact, illustrated in Figs. 3 and 4.

We now consider two trajectories, issuing, respectively from $P_{5}\left(X_{5}, Y_{5}\right)$ and $P_{6}\left(X_{6}, Y_{6}\right)$ as shown in Figure 12. By precisely the same arguments used in connection with the $T_{0}$-trajectory, $T_{5}$ passes above the origin and $T_{6}$ below it. Hence, there must exist a point $P^{* *}$ such that the trajectory $T^{* *}$ which issues from it passes through the origin without having a point of inflection. This is a modal line lying in the second quadrant. Since $x>0$ and $y<0$ everywhere along this modal line, the corresponding normal vibration is an out-of-phase mode. This establishes the following theorem.

Theorem 3. A system of two degrees of freedom whose equations of motion are (4) possesses an out-of-phase normal-mode-vibration in the sense of conditions (i) to (iv), if $U(x, y)$ is admissible in the sense of restrictions (a) to (c).

Hence, the basic theorem, stated earlier in the paper has been established.

Figure 13 shows a photograph of a graph of transversals, trajectories and the maximum equipotential line, done on an analog computer for the same problem as described in connection with Fig. 4. This illustration is similar to that of Fig. 11, except that it shows curves in the neighborhood of the out-of-phase mode. Again, it will be observed that the experimental results agree entirely with theoretical predictions.

Acknowledgments. Financial aid from the National Science Foundation which has supported this work is gratefully acknowledged. It is a pleasant duty to pay tribute here to Beverly Taskett's superior skill in the use of analog computers. The experimental results of Figs. 4, 11 and 13 are the fruits of her careful and painstaking work.

\section{REFERENCES}

1. R. M. Rosenberg, Normal modes of nonlinear dual-mode systems, J. Appl. Mech. 27 (1960) 263

2. R. M. Rosenberg, On normal vibrations of a general class of nonlinear dual-mode systems, J. Appl. Mech. 28 (1961) 275

3. R. M. Rosenberg, R. M. and C. S. Hsu, On the geometrization of normal vibrations of nonlinear systems having many degrees of freedom, Proceedings, IUTAM Symposium on Nonlinear Vibrations Kiev, 1963

4. R. M. Rosenberg, The normal modes of nonlinear n-degree-of-freedom systems, J. Appl. Mech. 29 (1962) 7

5. R. M. Rosenberg, and J. K. Kuo, Non-similar normal mode vibrations of nonlinear systems having two degrees of freedom, to be published in J. Appl. Mech.

6. See for instance N. Minorski, Nonlinear oscillations, D. Van Nostrand Company, Inc. 1962, pp. 9-26

7. H. Kauderer, Nichtlineare Mechanik, Springer Verlag, 1959, p. 599 